\title{
Temperature-Dependent Protein Backbone Dynamics from Auto- and Cross-Correlated NMR Relaxation Rates
}

\author{
L. Vugmeyster' and G. Bodenhausen ${ }^{1,2}$ \\ 'Institut des Sciences et Ingénierie Chimiques, Ecole Polytechnique Fédérale \\ de Lausanne, Lausanne, Switzerland \\ ${ }^{2}$ Département de Chimie, Associé au Centre National de la Recherche Scientifique, \\ Ecole Normale Supérieure, Paris, France
}

Received July 13, 2004

\begin{abstract}
The temperature dependence of nuclear magnetic resonance relaxation rates was investigated for the backbone of ${ }^{15} \mathrm{~N} /{ }^{13} \mathrm{C}$ labeled human ubiquitin in the temperature range of $20-50{ }^{\circ} \mathrm{C}$. The ${ }^{15} \mathrm{~N}$ autorelaxation rates give evidence that the potential energy functions for ${ }^{15} \mathrm{~N}-\mathrm{H}^{\mathrm{N}}$ bonds are not quadratic, in agreement with results for other proteins. Cross-correlation rates arising from correlated fluctuations of two ${ }^{15} \mathrm{~N}-\mathrm{H}^{\mathrm{N}}$ dipole-dipole interactions involving successive residues were obtained by the method of Pelupessy et al. (P. Pelupessy, S. Ravindranathan, G. Bodenhausen: J. Biomol. NMR $25,265-280,2003$ ). The results suggest the presence of slow internal motions at $50^{\circ} \mathrm{C}$.
\end{abstract}

\section{Introduction}

Dynamics of proteins occur on a wide range of time scales and many techniques have been devised to characterize internal motions [1-4]. Both correlated and uncorrelated motions are possible $[4,5]$. Correlated motions have been recently investigated with a variety of nuclear magnetic resonance (NMR) techniques [510]. It is interesting to study the change in internal dynamics under the influence of various perturbations. Temperature is one of the important factors, since it can provide a probe of many aspects such as protein stability, protein folding, and ligand binding [11-14], as well as an experimental means for investigating the energy landscape [15]. The temperature dependence of ${ }^{15} \mathrm{~N}$ autorelaxation rates in the backbone have been studied for a number of proteins [13, $14,16-25]$. The longitudinal and transverse relaxation rates and heteronuclear Overhauser effects [26] are often analyzed by the model-free formalism of Lipari and Szabo [27] and its extension by Clore and co-workers [28]. On the basis of the temperature dependence of the autorelaxation rates, it has been suggested that backbone dynamics play little role in heat capacity differences between folded 
and unfolded states of proteins $[14,16,17]$. Zuiderweg and co-workers [24] have compared the temperature dependence of ${ }^{15} \mathrm{~N}-{ }^{1} \mathrm{H}$ and ${ }^{13} \mathrm{C}^{\prime}-{ }^{13} \mathrm{C}^{\alpha}$ reorientational dynamics for binase and ubiquitin. Their results give a strong indication that some backbone motions activated at room temperature are not sensed by the ${ }^{15} \mathrm{~N}$ ${ }^{\prime} \mathrm{H}$ vectors. This is in agreement with recent multiple quantum experiments at room temperature involving ${ }^{13} \mathrm{C}^{\prime}$ and ${ }^{15} \mathrm{~N}$ nuclei [29].

In this work we analyze the temperature dependence of cross-correlated relaxation rates arising from correlated fluctuations of two $\mathrm{N}-\mathrm{H}^{\mathrm{N}}$ dipole-dipole interactions involving successive residues in a ${ }^{15} \mathrm{~N} /{ }^{13} \mathrm{C}$ labeled sample of human ubiquitin in the temperature range of $20-50{ }^{\circ} \mathrm{C}$. For the determination of the cross-correlated relaxation rates we used the method developed by Pelupessy et al. [10]. The ${ }^{15} \mathrm{~N}$ autorelaxation rates were obtained under the same conditions. Ubiquitin is an extremely thermostable protein, the midpoint of temperature-induced unfolding being at $91{ }^{\circ} \mathrm{C}$ [30]. Zuiderweg and co-workers [24] obtained autorelaxation rates for ubiquitin in the range of $5-45{ }^{\circ} \mathrm{C}$. The temperature dependence of hydrogen bond strengths have been analysed for ${ }^{15} \mathrm{~N} /{ }^{13} \mathrm{C} /{ }^{2} \mathrm{D}$ ubiquitin at $\mathrm{pH} 6.5$ in the range of $25-55^{\circ} \mathrm{C}$ [18].

\section{Cross-Correlation Spectral Densities}

In this section we give a brief outline of the model that is used to derive crosscorrelated spectral density functions. The details of the theory have been presented elsewhere [31]. We consider correlated fluctuations of two interactions $\mathbf{u}$ and $\mathbf{v}$. The model is valid for dipole-dipole interactions between nuclei that are at a fixed distance from each other, and for chemical shift anisotropy interactions described by tensors that are axially symmetric with components which are independent of internal motions. The dynamics of each interaction $\mathbf{u}$ will be described by three parameters, i.e., two local correlation times $\tau_{u}^{\mathrm{f}}$ and $\tau_{u}^{\mathrm{s}}$ corresponding to fast and slow internal motions, in addition to the isotropic global correlation time $\tau_{\mathrm{c}}$, which characterizes the global tumbling of the molecule. In this work we consider cases for which $\tau_{u}^{\mathrm{f}} \ll \tau_{\mathrm{c}} \ll \tau_{u}^{\mathrm{s}}$. In an earlier work [31] more general cases have been considered.

The overall cross-correlation function $C_{u v}(t)$ can be decomposed into contributions due to overall and internal motions [27]:

$$
C_{u v}(t)=C^{\text {glob }}(t) C_{u v}^{\text {int }}(t)
$$

A two-step model is presented for the cross-correlation function of the internal motions $C_{u v}^{\text {int }}(t)$. In a first step, the cross-correlation function reaches a quasi-equilibrium state $C_{u \nu}^{\mathrm{int}, q}(t)$, corresponding to the equilibrium with respect to the fast fluctuations with a characteristic time-constant $\tau_{u}^{\mathrm{f}}$. In a second step, the quasiequilibrium state converges to the true equilibrium, characterized by the crosscorrelated order parameter $S_{u v}^{2}=C_{u v}^{\text {inteq }}$ with a characteristic time-constant $\tau_{u}^{\mathrm{s}}$ 


$$
\begin{gathered}
\frac{\mathrm{d} C_{u \nu}^{\mathrm{int}}(t)}{\mathrm{d} t}=-\frac{1}{\tau_{u}^{\mathrm{f}}}\left(C_{u v}^{\mathrm{int}}(t)-C_{u \nu}^{\mathrm{int}, \mathrm{q}}(t)\right), \\
\frac{\mathrm{d} C_{u \nu}^{\mathrm{int}, \mathrm{q}}(t)}{\mathrm{d} t}=-\frac{1}{\tau_{u}^{\mathrm{s}}}\left(C_{u \nu}^{\mathrm{int}, \mathrm{q}}(t)-C_{u \nu}^{\mathrm{int}, \mathrm{eq}}\right) .
\end{gathered}
$$

Fast internal motions will be considered to be independent and axially symmetric for the two interactions $\mathbf{u}$ and $\mathbf{v}$. Since $\tau_{u}^{\mathrm{f}} \ll \tau_{u}^{\mathrm{s}}$, the system rapidly converges to the quasi-equilibrium state. Slow motions can be described in terms of mobility of the quasi-equilibrium vectors $\mathbf{u}^{q}$ and $\mathbf{v}^{q}$.

Three scenarios are considered for slow internal motions: (i) uncorrelated fluctuations of $\mathbf{u}^{\mathrm{q}}$ and $\mathbf{v}^{\mathrm{q}}$; (ii) a "rigid-fragment" case corresponding to synchronous fluctuations of $\mathbf{u}^{\mathrm{q}}$ and $\mathbf{v}^{\mathrm{q}}$ with a constant angle $\theta_{u v}^{\mathrm{q}}$ between them; (iii) partial correlation of the motions of $\mathbf{u}^{\mathrm{q}}$ and $\mathbf{v}^{\mathrm{q}}$. Figure 1 depicts the rigid-fragment (top) and uncorrelated cases (bottom). Fast motions of the two vectors $\mathbf{u}$ and $\mathbf{v}$ are represented by cones. The slow motions of $\mathbf{u}^{\mathrm{q}}$ and $\mathbf{v}^{\mathrm{q}}$ are represented by reorientations of the axes of the cones. Using the two-step model described above, expressions for the spectral densities can be derived for cases i-iii.

(i) For uncorrelated fluctuations,

$$
J_{u \nu}(\omega)=\frac{2}{5} S_{u \nu}^{2} \frac{\tau_{c}}{1+\omega^{2} \tau_{c}^{2}} .
$$

If we assume that the slow fluctuations are axially symmetric [31], the crosscorrelated order parameter can be decomposed into a product of autocorrelated

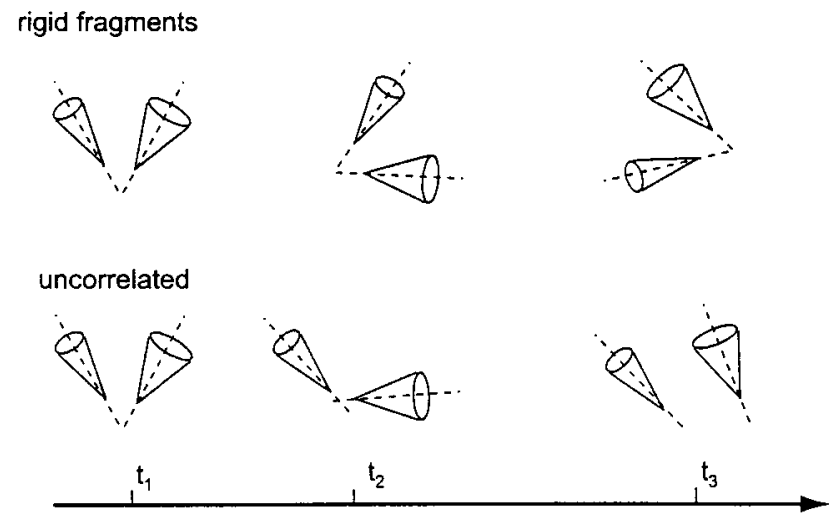

Fig. 1. Illustration of two extremes of slow internal motions. Fast axially symmetric uncorrelated motions of the vectors $\mathbf{u}$ and $\mathbf{v}$ are represented by two cones. Time is represented along the horizontal axis with three different time points marked as $t_{1}, t_{2}$, and $t_{3}$. The axes of the cones, which are depicted by dashed lines, represent the quasi-equilibrium vectors $\mathbf{u}^{9}$ and $\mathbf{v}^{q}$. The top figure represents the rigidfragment case, where the angle $\theta_{u v}^{\mathrm{a}}$ between the axes is fixed, and the bottom figure represents the uncorrelated case, where the axes of the two cones are independent of each other. 
order parameters $S_{u u}^{\mathrm{f}}$ and $S_{v v}^{\mathrm{f}}$ due to fast motions, and order parameters $S_{u u}^{\mathrm{s}}$ and $S_{v v}^{\mathrm{s}}$ due to slow motions

$$
S_{u v}^{2}=S_{u u}^{\mathrm{f}} S_{v v}^{\mathrm{f}} S_{u u}^{\mathrm{s}} S_{v v}^{\mathrm{s}} P_{2}\left(\cos \theta_{u v}^{\mathrm{eq}}\right),
$$

where $\theta_{u v}^{\text {eq }}$ is the angle between the equilibrium vectors $\mathbf{u}^{\text {eq }}$ and $v^{\text {eq }}$ and $P_{2}$ is the Legendre polynomial. This results in the following expression for the spectral density function:

$$
J_{u v}(\omega)=\frac{2}{5} S_{u u}^{\mathrm{f}} S_{v v}^{\mathrm{f}} S_{u u}^{\mathrm{s}} S_{v v}^{\mathrm{s}} P_{2}\left(\cos \theta_{u v}^{\mathrm{eq}}\right) \frac{\tau_{\mathrm{c}}}{1+\omega^{2} \tau_{\mathrm{c}}^{2}} .
$$

(ii) For a rigid fragment,

$$
J_{u v}^{\text {rigid }}(\omega)=\frac{2}{5} S_{u u}^{\mathrm{f}} S_{v v}^{\mathrm{f}} P_{2}\left(\cos \theta_{u \nu}^{\mathrm{q}}\right) \frac{\tau_{c}}{1+\omega^{2} \tau_{\mathrm{c}}^{2}},
$$

where $\theta_{u v}^{\mathrm{q}}$ is the angle between $\mathbf{u}^{\mathrm{q}}$ and $\mathbf{v}^{\mathrm{q}}$, which is a constant in this case, so that $\left\langle\theta_{u v}^{q}\right\rangle=\theta_{u v}^{q}$. Equation (7) can be compared with Eq. (6), which is valid in the absence of correlations. A remarkable feature of the spectral density in these two distinct regimes is the proportionality of $J_{u v}(\omega)$ to either $P_{2}\left(\cos \theta_{u v}^{\text {eq }}\right)$ for the uncorrelated case or to $P_{2}\left(\cos \theta_{u v}^{\mathrm{q}}\right)$ for a rigid fragment. Neither of these proportionalities will however apply to the case of partial correlations. Note that in general the average angle $\left\langle\theta_{u v}^{q}\right\rangle$ between the two vectors $\mathbf{u}^{q}$ and $v^{q}$ is not equal to the angle $\theta_{u v}^{\text {eq }}$. The angle $\theta_{u \nu}^{\text {eq }}$ can be estimated from X-ray or NMR structures.

(iii) For partial correlations,

$$
J_{u v}(\omega)=\frac{2}{5} C_{u v}^{\mathrm{int}, \mathrm{q}}(0) \frac{\tau_{\mathrm{c}}}{1+\omega^{2} \tau_{\mathrm{c}}^{2}}=\frac{2}{5} S_{u u}^{\mathrm{f}} S_{v v}^{\mathrm{f}}\left\langle P_{2}\left(\cos \theta_{u v}^{\mathrm{q}}\right)\right\rangle \frac{\tau_{\mathrm{c}}}{1+\omega^{2} \tau_{\mathrm{c}}^{2}} .
$$

As can be seen from the above equations, slow motions enter into the crosscorrelation spectral density only if they are partly or totally uncorrelated. For the rigid-fragment case there is neither distribution nor time dependence of $\theta_{u v}^{q}$. The case of autocorrelation may be treated as a special case of a rigid fragment with $\theta_{u v}^{q}=0$. Motions that are slower than the global tumbling of the molecule do not enter into the autocorrelation spectral densities [28]. Using the two-step model described above, the spectral density for the autocorrelation case can be written as

$$
J_{u v}(\omega)=\frac{2 \pi}{5}\left[\left(\tilde{S}_{u u}^{\mathrm{f}}\right)^{2} \frac{\tau_{\mathrm{c}}}{1+\omega^{2} \tau_{\mathrm{c}}^{2}}+\left(1-\left(\tilde{S}_{u u}^{\mathrm{f}}\right)^{2}\right) \frac{\tau_{u}^{\mathrm{f}^{\prime}}}{1+\omega^{2} \tau_{u}^{\mathrm{f}^{\mathrm{f}^{\prime}}}}\right],
$$

where

$$
\left(\tau_{u}^{\mathbf{f}^{\prime}}\right)^{-1}=\left(\tau_{u}^{\mathbf{f}}\right)^{-1}+\left(\tau_{\mathrm{c}}\right)^{-1}
$$

using the extended Lipari-Szabo order parameter $\left(\tilde{S}^{\mathrm{f}}\right)^{2}$ (which we call $\left(\tilde{S}_{u u}^{\mathrm{f}}\right)^{2}$ to avoid ambiguities of notation) describing the fast dynamics of the vector $\mathbf{u}$. This 
relates to our definition based on the two-step model for the correlation function of the internal motions in the following manner:

with

$$
\left(\tilde{S}_{u u}^{\mathrm{f}}\right)^{2}=\alpha\left(S_{u u}^{\mathrm{f}}\right)^{2}+(1-\alpha)\left(S_{u u}\right)^{2}
$$

$$
\alpha=\frac{\tau_{u}^{\mathrm{s}}}{\tau_{u}^{\mathrm{s}}-\tau_{u}^{\mathrm{f}}},
$$

where $\left(S_{u u}\right)^{2}$ is the total autocorrelation order parameter including both slow and fast motions. Thus, the approach of Clore and coworkers [28] gives a higher estimate of the order parameter corresponding to fast fluctuations than our model. The cause of this discrepancy is that Clore et al. considered slow and fast motions as two independent processes. However, the correction is very small, since $\alpha$ is close to unity for $\tau_{u}^{\mathrm{f}} \ll \tau_{u}^{\mathrm{s}}$.

\section{Results and Discussion}

\subsection{Autocorrelation}

Figure 2 displays the generalized order parameters for fast internal motions, which were obtained from $R_{1}, R_{2}$, and heteronuclear ${ }^{15} \mathrm{~N}\left\{{ }^{1} \mathrm{H}\right\}$ NOE data assuming axially symmetric diffusion tensors. Table 1 shows the values of $\tau_{\mathrm{c}}$ and ratios of $D_{\|} / D_{\perp}$ of the components of the diffusion tensor at each temperature. The values of Table 1 at $31.1^{\circ} \mathrm{C}$ agree well with the previously reported values for ubiquitin under similar conditions [32]. The values of $\left(S_{u u}^{\text {f,exp }}\right)^{2}$ do not vary strongly along the protein backbone with the exception of the $\mathrm{C}$ terminus. Residues that belong to the loop between the first two $\beta$ sheets and the beginning of the second $\beta$ sheet (T7, L8, T9, G10, K11, T12) as well as $\mathrm{I} 36$ and Q62, which are also located in loop regions, tend to have lower values of $\left(S_{u u}^{\text {fexp }}\right)^{2}$.

The temperature dependence of the autorelaxation rates can be characterized by the slopes of the plots of $\ln \left(1-S_{u u}^{\mathrm{f}, \mathrm{exp}}\right)$ versus $\ln T$.

$$
\Lambda=\frac{\mathrm{d} \ln \left(1-S_{u u}^{\mathrm{fexp}}\right)}{\mathrm{d} \ln T}
$$

where $T$ is the temperature. The parameter $A$ is closely related to the characteristic temperature, $T^{*}=3 T /\left[2\left(1-S_{u u}^{\text {f,exp }}\right) \Lambda\right]$, which has been used for similar purposes $[14,17,19,20]$. Values of $A>1$ imply that the potential energy function for the bond vector is not quadratic [16]. Values of $A>1$ were observed for villin headpiece helical subdomain [16], B1 domain of streptococcal protein G [14], and ribonuclease $H$ [17].

The parameters $\Lambda$ were determined only for residues where data at all four temperatures could be obtained. Residues with errors in excess of $50 \%$ were discarded. The values of $A$ are listed in Table 2. The large errors are the result of 

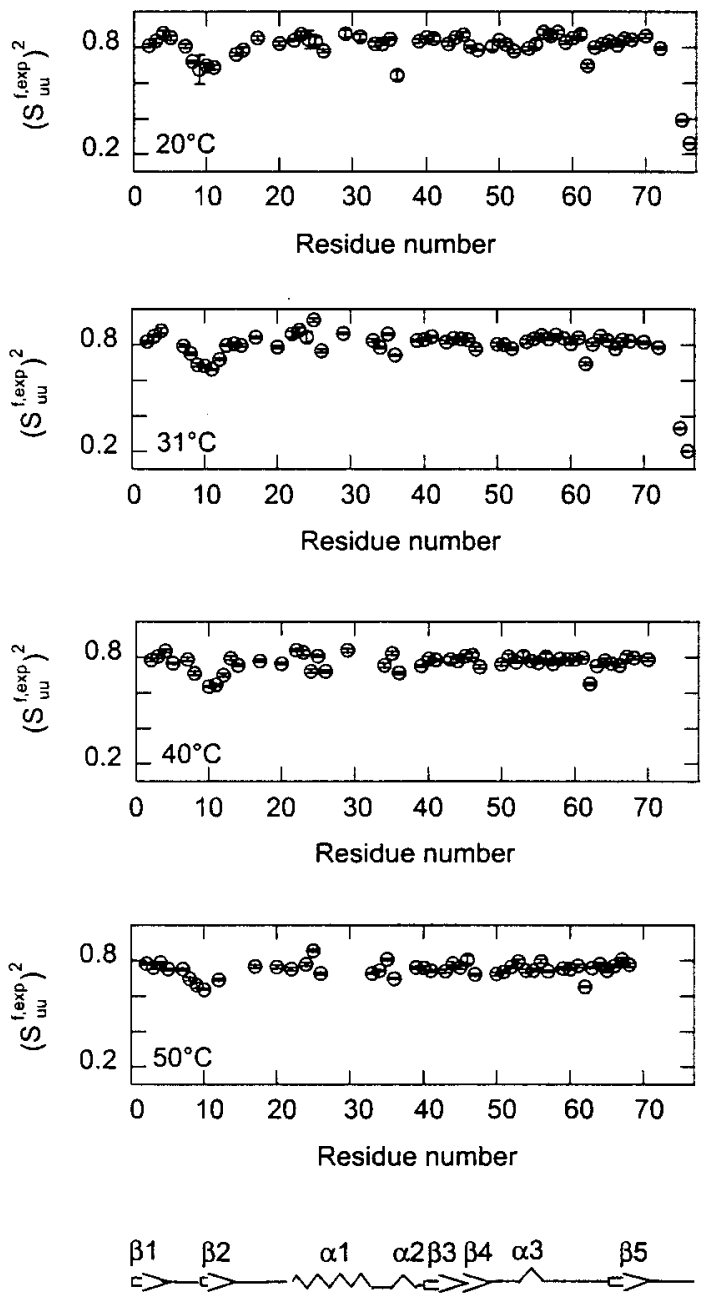

Fig. 2. Experimentally determined ${ }^{15} \mathrm{~N}$ autocorrelated order parameters $\left(S_{u u}^{\text {f.exp }}\right)^{2}$ associated with fast motions of amide bonds in ubiquitin determined at four temperatures by the Lipari-Szabo procedure explained in the text.

Table 1. Parameters characterizing anisotropic overall motion.

\begin{tabular}{ccc}
\hline$T\left({ }^{\circ} \mathrm{C}\right)$ & $D_{11} / D_{\perp}$ & $\tau_{\mathrm{c}}(\mathrm{ns})$ \\
\hline 20.0 & $1.28 \pm 0.04$ & $4.74 \pm 0.03$ \\
31.1 & $1.18 \pm 0.04$ & $3.81 \pm 0.02$ \\
40.5 & $1.05 \pm 0.06$ & $3.43 \pm 0.02$ \\
50.2 & $0.81 \pm 0.04$ & $2.89 \pm 0.02$ \\
\hline
\end{tabular}


Table 2. Values of $A$.

\begin{tabular}{llllll}
\hline Residue & $A$ & Error & Residue & $\Lambda$ & Error \\
\hline I3 & 5.6 & 2.3 & G47 & 2.8 & 0.8 \\
T7 & 3.1 & 1.4 & L50 & 5.8 & 1.4 \\
G10 & 1.8 & 0.7 & E51 & 4.4 & 1.7 \\
V17 & 5.7 & 2.3 & R54 & 3.0 & 1.8 \\
T22 & 5.1 & 1.7 & T55 & 4.9 & 2.3 \\
V26 & 2.5 & 1.2 & S57 & 6.5 & 2.3 \\
E34 & 3.6 & 1.2 & Y59 & 5.4 & 1.6 \\
D39 & 4.4 & 1.8 & N60 & 5.0 & 1.8 \\
Q40 & 6.0 & 2.0 & I61 & 6.1 & 3.2 \\
Q41 & 7.6 & 2.1 & Q62 & 1.6 & 0.7 \\
L43 & 4.8 & 1.9 & S65 & 5.9 & 1.8 \\
I44 & 5.1 & 2.4 & H68 & 4.0 & 1.9 \\
F45 & 6.4 & 2.7 & & & \\
\hline
\end{tabular}

the fact that the majority of the residues had to be fitted with different motional models at the four temperatures. The temperature dependence of the autocorrelation rates shows that $\Lambda>1$ for all residues where it could be determined. The average value of $A$ in Table 2 is 4.7 with a standard deviation of 1.5 . This indicates that the potential function for the fast motions of the $\mathrm{N}-\mathrm{H}^{\mathrm{N}}$ bonds is not quadratic, in agreement with data for other proteins.

\subsection{Cross-correlation}

To simplify the analysis of the cross-correlation rates, the assumption of isotropic overall tumbling was used [32-36], although Table 1 shows that the diffusion anisotropy can be as large as $D_{\| \mid} / D_{\perp}=1.28 \pm 0.04$ at $20{ }^{\circ} \mathrm{C}$. It has been shown that the generalized Lipari-Szabo order parameters for fast motions obtained with axial or isotropic diffusion tensors are very similar, provided that the same motional models are used [32]. For the analysis of the cross-correlation rates we used the fast generalized order parameters described in the previous section.

For the determination of $\mathrm{NH} / \mathrm{NH}$ dipole-dipole cross-correlation rates, the $\mathrm{DQ}$ version of the experiment described by Pelupessy and coworkers [10] was performed. This avoids the effects of cross-relaxation involving the two amide protons, in contrast to the experiments where both DQ and ZQ signals are recorded. The experiment is designed to detect the interconversion between $\left\langle 2 N_{+}^{u} N_{+}^{v}\right\rangle$ and $\left\langle 8 N_{+}^{u} H_{z}^{u} N_{+}^{v} H_{z}^{v}\right\rangle$ operators. The main mechanism of interconversion is due to correlated fluctuations of the dipolar interactions $N^{u} H^{u}$ and $N^{v} H^{v}$ were $u$ and $v$ denote adjacent residues. The rate is obtained from the ratio of the intensities $I_{\text {cross }}$ and $I_{\mathrm{ref}}$, which are proportional to the expectation values $\left\langle 8 N_{+}^{u} H_{Z}^{u} N_{+}^{v} H_{Z}^{v}\right\rangle$ and $\left\langle 2 N_{+}^{u} N_{+}^{\nu}\right\rangle$ operators, respectively: 


$$
\frac{I_{\text {cross }}(T)}{I_{\text {ref }}(T)}=\tanh \left(R_{u v}^{\exp } T\right) .
$$

Hence, one can obtain experimental relaxation rates

$$
R_{u v}^{\text {exp }}=\frac{1}{T} \tanh ^{-1} \frac{I_{\text {cross }}(T)}{I_{\text {ref }}(T)} .
$$

At $31{ }^{\circ} \mathrm{C}$ there is good agreement with the rates previously reported for ubiquitin at $30^{\circ} \mathrm{C}[10]$.

We assume that the slow motions are uncorrelated, which corresponds to the spectral density of Eq. (6). Even though on general grounds there is no reason to believe that slow motions are uncorrelated and axially symmetric for all residues in a protein, nevertheless we will use this simple assumption since it allows us to make an estimate of the order parameters of slow motions. We shall also discuss deviations from this assumption. From the relationship between the rates and the spectral densities (retaining only the main contribution at zero frequency, $J(\omega=0)$ ) the theoretical rate is given by

$$
R_{u v}^{\text {theor }}=\left(\frac{\mu_{0} \hbar \gamma_{\mathrm{H}} \gamma_{\mathrm{N}}}{4 \pi r_{\mathrm{NH}}^{3}}\right)^{2} J(0)=\left(\frac{\mu_{0} \hbar \gamma_{\mathrm{H}} \gamma_{\mathrm{N}}}{4 \pi r_{\mathrm{NH}}^{3}}\right)^{2} S_{u u}^{\mathrm{f}} S_{v v}^{\mathrm{f}} S_{u u}^{\mathrm{s}} S_{v v}^{\mathrm{s}} P_{2}\left(\cos \theta_{u v}^{\mathrm{eq}}\right) \frac{2 \tau_{\mathrm{c}}}{5},
$$

where all symbols have their usual meaning. If there is neither fast nor slow local motion, i.e., if $S_{u u}^{\mathrm{f}}=S_{v v}^{\mathrm{f}}=S_{u u}^{\mathrm{s}}=S_{v v}^{\mathrm{s}}=1$, the cross-correlated rate is

$$
R_{u v}^{\text {rigid }}=\left(\frac{\mu_{0} \hbar \gamma_{\mathrm{H}} \gamma_{\mathrm{N}}}{4 \pi r_{\mathrm{NH}}^{3}}\right)^{2} P_{2}\left(\cos \theta_{u v}^{\mathrm{eq}}\right) \frac{2 \tau_{\mathrm{c}}}{5} .
$$

This function is represented by curves in Fig. 3. The correlation times $\tau_{\mathrm{c}}$ that were obtained at different temperatures are given in Table 1.

Pelupessy et al. [10] defined a "heuristic" order parameter:

$$
S_{u v}^{2(\text { heur })}=\frac{R_{u v}^{\exp }}{R_{u v}^{\text {rigid }}} .
$$

By contrast, we shall consider the ratio of experimentally determined parameters

$$
\tilde{R}_{u v}^{\exp }=\frac{R_{u v}^{\exp }}{S_{u u}^{\mathrm{f}, \exp } S_{w}^{\mathrm{f}, \exp }} .
$$

The values of $\tilde{R}_{u u}^{\text {exp }}$ are shown in Fig. 3. These can be compared with a ratio that follows from Eq. (16):

$$
\tilde{R}_{u v}^{\text {theor }}=\frac{R_{u v}^{\text {theor }}}{S_{u u}^{\mathrm{f}} S_{v v}^{\mathrm{f}}}=\left(\frac{\mu_{0} \hbar \gamma_{\mathrm{H}} \gamma_{\mathrm{N}}}{4 \pi r_{\mathrm{NH}}^{3}}\right)^{2} S_{u u}^{\mathrm{s}} S_{v v}^{\mathrm{s}} P_{2}\left(\cos \theta_{u v}^{\mathrm{eq}}\right) \frac{2 \tau_{\mathrm{c}}}{5} .
$$

There is a number of residues that exhibit deviations of $\tilde{R}_{u u}^{\text {exp }}$ from $R_{u v}^{\text {rigid }}$ (given by curves) at all temperatures. The data have been acquired in the temperature range 

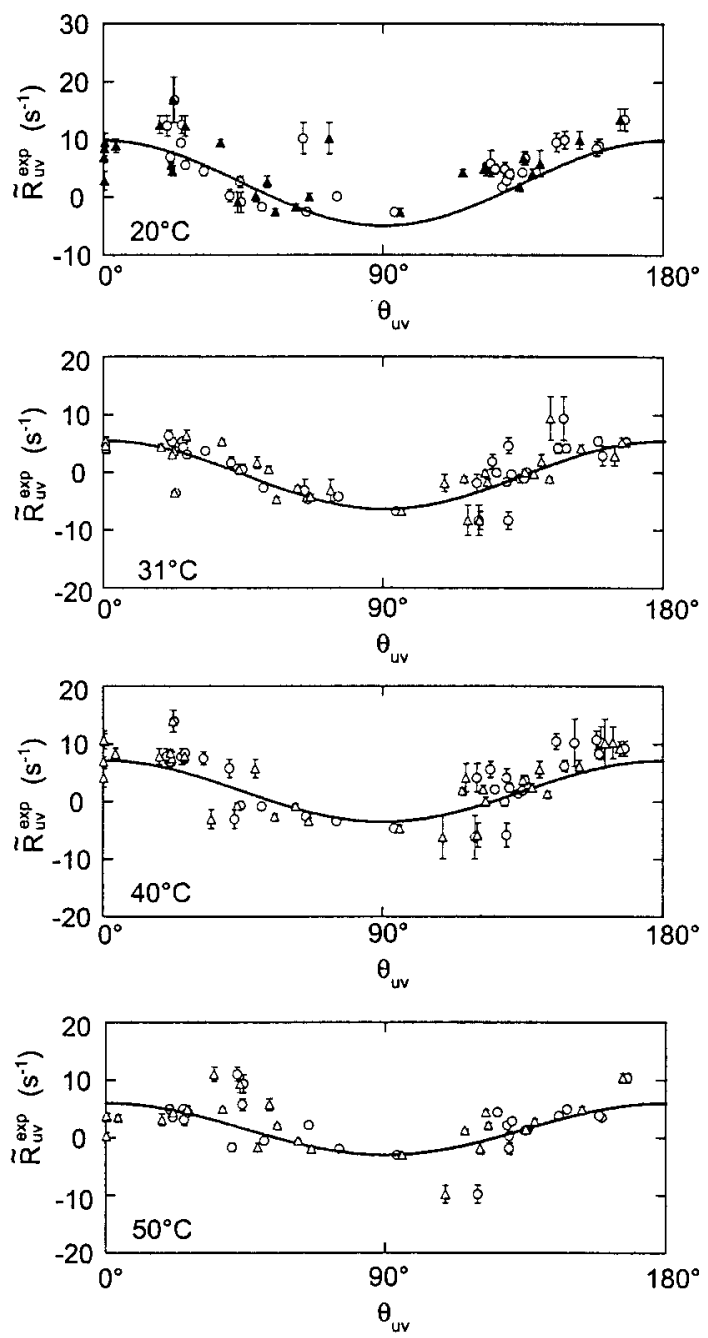

Fig. 3. Ratios $\tilde{R}_{u \nu}^{\text {exp }}$ of the cross-correlation rate $R_{u \psi}^{\exp }$ determined from the relaxation of double quantum coherences involving two ${ }^{15} \mathrm{~N}$ nuclei in consecutive amino acids divided by the square root of the product of the fast autorelaxation order parameters shown in Fig. 2, as defined in Eq. (19). The rates $\tilde{R}_{u v}^{\text {exp }}$ are plotted versus $\theta_{u v}$ at four temperatures. The angles $\theta_{u v}$ are taken either from the NMR structure $(O)$ or X-ray structure $(\Delta)$. The curves depict the function $R_{u v}^{\text {rigid }}$ of Eq. (17).

where the protein is at least $99 \%$ in a folded conformation so that we may assume that it has the same structure at all temperatures. Both NMR and X-ray structures were originally determined at $30^{\circ} \mathrm{C}[37,38]$. If the angles $\theta_{u v}^{\text {eq }}$ are correctly given by the NMR structure, the deviations from the $R_{u v}^{\text {rigid }}$ curve are indicative of the presence of slow motions. These motions must have at least partially uncorrelated character, otherwise they would not be detectable, as shown above. 
The results depend on whether NMR or X-ray structures have been used to determine $\theta_{u v}$. The values of $\tilde{R}_{u u}^{\exp }$ tend to be lower when the X-ray structure is used.

Clearly, both $\tilde{R}_{t a t}^{\text {exp }}$ and $\tilde{R}_{t \omega}^{\text {theor }}$ depend on a structural factor embodied by the Legendre polynomial in Eq. (20). To separate this structural contribution, we define
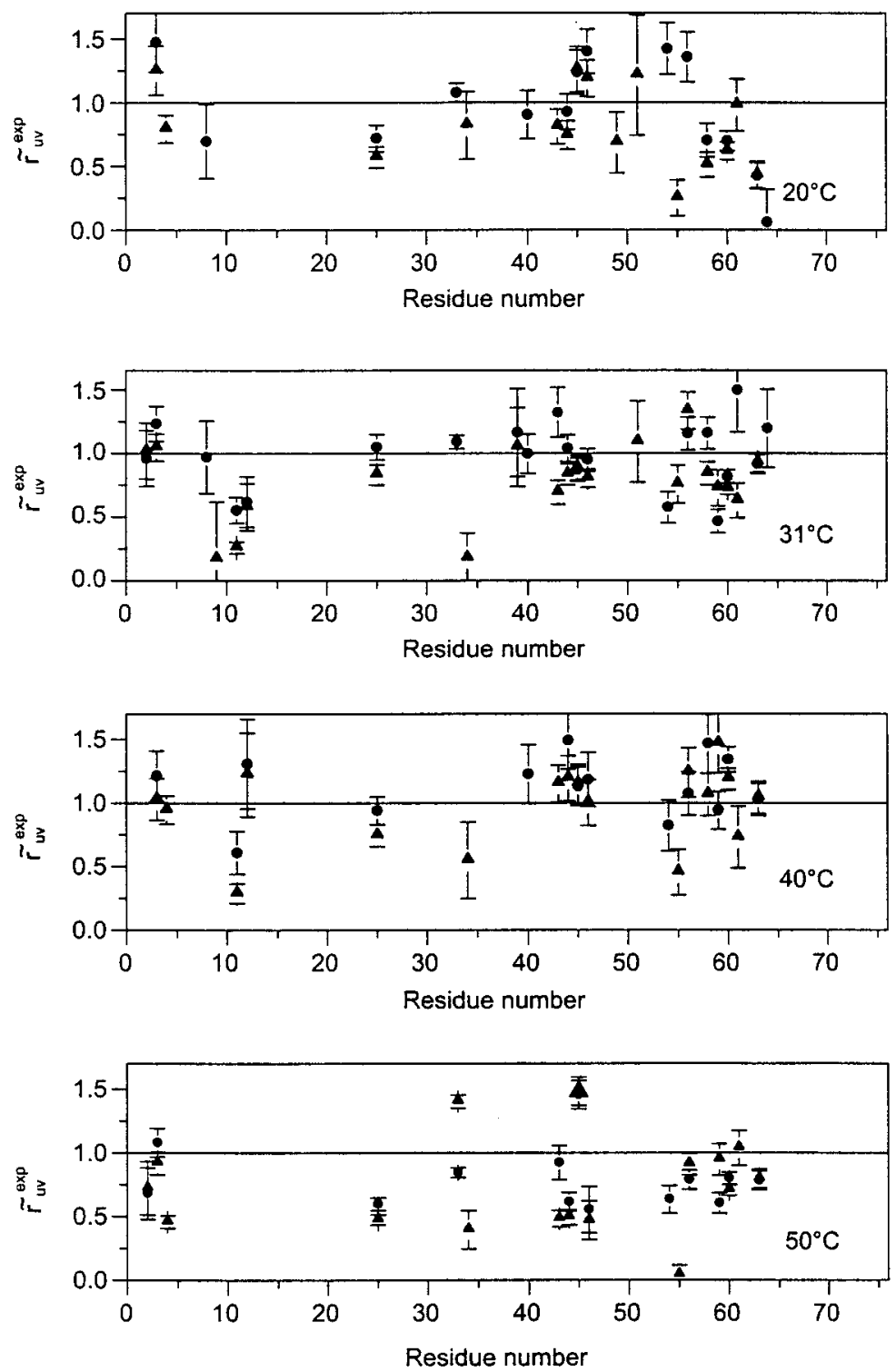

Fig. 4. Plots of $\tilde{r}_{u \nu}^{\text {exp }}$ defined in Eq. (22) versus residue number at four temperatures with angles taken from either NMR structure $(\bullet)$ or X-ray structure $(\mathbf{A})$. 


$$
\tilde{r}_{u v}^{\text {theor }}=\left(\frac{4 \pi r_{\mathrm{NH}}^{3}}{\mu_{0} \hbar \gamma_{\mathrm{H}} \gamma_{\mathrm{N}}}\right)^{2} \frac{5}{2 \tau_{\mathrm{c}}} \frac{\tilde{R}_{u v}^{\text {theor }}}{P_{2}\left(\cos \theta_{u v}^{e q}\right)}=S_{u u}^{\mathrm{s}} S_{v v}^{\mathrm{s}},
$$

which is equal to 1 in the absence of slow motions. The angles $\theta_{u \nu}^{\text {eq }}$ can be taken either from NMR or X-ray structures $[37,38]$. Likewise, one can normalize the experimentally determined ratios

$$
\tilde{r}_{u v}^{\exp }=\left(\frac{4 \pi r_{\mathrm{NH}}^{3}}{\mu_{0} \hbar \gamma_{\mathrm{H}} \gamma_{\mathrm{N}}}\right)^{2} \frac{5}{2 \tau_{\mathrm{c}}} \frac{\tilde{R}_{u v}^{\exp }}{P_{2}\left(\cos \theta_{u v}^{\mathrm{eq}}\right)} .
$$

Comparison of Eq. (21) and Eq. (22) shows that $\tilde{r}_{u v}^{\text {exp }}$ can be interpreted as a measure of slow internal motions

$$
\tilde{r}_{u v}^{\exp }=S_{u u}^{s, \exp } S_{v v}^{s, \exp }
$$

These normalized experimental rates are plotted in Fig. 4. Residues for which there were no fast autocorrelation order parameters available for either $\mathbf{u}$ or $\mathbf{v}$ vectors had to be discarded from the analysis. The horizontal lines represent the case of $\tilde{r}_{u v}^{\exp }=1$, i.e., the absence of slow motions.

The expression for slow motions of Eq. (21) must obey the inequality $0<\tilde{r}_{u v}^{\text {theor }}<1$. When the experimental ratios $\tilde{r}_{u v}^{\text {exp }}$ are not contained in the interval $0<\tilde{r}_{u v}^{\exp }<1$, this can be due to deviations from the assumptions of uncorrelated axially symmetric motions and/or poor estimates of $\theta_{u v}^{\text {eq }}$ from either NMR or X-ray structures. Order parameters smaller than one indicate the presence of slow motions. At $50.2{ }^{\circ} \mathrm{C}$ there are many more residues that are below the horizontal line, which indicates that slow motions are more pronounced at this temperature.

\section{Experimental}

The experiments were performed on ${ }^{15} \mathrm{~N} /{ }^{13} \mathrm{C}$ labeled human ubiquitin obtained commercially (VLI). The protein was dissolved in $200 \mu \mathrm{l}$ of $90 \% \mathrm{H}_{2} \mathrm{O}-10 \% \mathrm{D}_{2} \mathrm{O}$ to a concentration of $1.4 \mathrm{mM}$ with phosphate buffer and the $\mathrm{pH}$ was adjusted to 4.5. A Shigemi tube made of susceptibility-matched glass was used.

\subsection{Autocorrelation Data}

The NMR autocorrelation rates were measured at 20.0,31.1, 40.5 and $50.2{ }^{\circ} \mathrm{C}$ on a Bruker DRX spectrometer operating at a Larmor frequency of $400.11 \mathrm{MHz}$, equipped with a triple resonance TBI probe with triple axes gradients. The sample temperature was calibrated using $100 \%$ methanol and $100 \%$ ethylene glycol standards. ${ }^{15} \mathrm{~N} R_{1}, R_{2}$, and heteronuclear NOE experiments were collected by previously described protocols $[26,39] . R_{2}$ experiments used CPMG pulse trains with a spac- 
ing of $1 \mathrm{~ms}$ and the States-TPPI quadrature detection scheme [40]. $R_{1}$ and NOE experiments used the sensitivity enhancement scheme [41, 42]. The experimental points were recorded for $t=0.01,0.08,1.3,0.14,1.17,0.25,1.0,0.38,0.70,0.55$, $0.85,0.01 \mathrm{~s}$ for $R_{1}$ and $t=0.0086,0.0172,0.2414,0.0345,0.2069,0.0604,0.1811$, $0.0948,0.1724,0.1207,0.1466,0.0172,0.20692 \mathrm{~s}$ for $R_{2}$. The NOE experiments were obtained from pairs of spectra with and without proton saturation during the recycle delay. The saturation period was $4 \mathrm{~s}$ in the NOE and $5 \mathrm{~s}$ in non-NOE experiments. Each NOE experiment was repeated three times. $1024 \times 100$ complex points were collected with spectral widths of 13 and $25 \mathrm{ppm}$ in the ${ }^{1} \mathrm{H}$ and ${ }^{15} \mathrm{~N}$ dimensions, respectively. The spectra were processed using NMRPipe software [43] with scripts available at www.palmer.hs.columbia.edu. A sinebell window function was used in both dimensions. Signal intensities were taken for nonoverlapping peaks only. $R_{1}$ and $R_{2}$ values were determined by nonlinear least-square fitting of the experimental data to a two-parameter monoexponential fit. Uncertainties were obtained using jack-knife simulations [44] for $R_{1}$ and $R_{2}$ experiments. For the heteronuclear NOE, the uncertainties were obtained from three measurements. $R_{1}$ and $R_{2}$ data were used to obtain axially symmetric diffusion tensors at four temperatures. The C-terminal residues were not included in this analysis as well as the residues that do not lie within two standard deviations of the average values of $F=\left(\left\langle T_{2}\right\rangle-T_{2}\right) /\left\langle T_{2}\right\rangle-\left(\left\langle T_{1}\right\rangle-T_{1}\right) /\left\langle T_{1}\right\rangle$, where $T_{1}=1 / R_{1}$ and $T_{2}=1 / R_{2}$ [32]. The values of the diffusion tensors where used along with $R_{1}, R_{2}$, and NOE data to obtain model-free Lipari-Szabo parameters. This was performed using ModelFree and Quadratic diffusion programs available at the web page indicated above. The selection of the motional model for each residue was done using the procedure described by Mandel et al. [45]. Most residues were fitted with model 1 at 20 and $31 .{ }^{\circ} \mathrm{C}$ and with model 2 at 40.5 and $50.2{ }^{\circ} \mathrm{C}$. The following residues required model 3: $\mathrm{N} 25$, T55 at $20^{\circ} \mathrm{C}$; D52, G53 at $40.5{ }^{\circ} \mathrm{C}$; A46, G53 at $50.2{ }^{\circ} \mathrm{C}$. The following residues could not be fitted with any motional model: $\mathrm{T} 12, \mathrm{I} 13$ at $20^{\circ} \mathrm{C}$; $\mathrm{T} 9, \mathrm{~L} 15, \mathrm{~K} 33, \mathrm{R} 72, \mathrm{G} 75, \mathrm{G} 76$ at $40.5^{\circ} \mathrm{C}$; and $\mathrm{K} 11, \mathrm{~N} 25, \mathrm{~K} 29$, D58, V70, G75, G76 at $50.2{ }^{\circ} \mathrm{C}$.

\subsection{Cross-correlated Data}

The NMR cross-correlation rates were measured on a Bruker DMX spectrometer operating at $600.13 \mathrm{MHz}$ and equipped with a triple resonance TBI probe with triple axes gradients. The measurements were perfomed at the same temperatures as the autocorrelation data. The double quantum dipole-dipole/dipole-dipole $\mathrm{NH} / \mathrm{NH}$ cross-correlated experiments were performed as described by Pelupessy et al. [10]. The experiments were performed with a single relaxation delay $T=43 \mathrm{~ms}$. Two-dimensional spectra were acquired in an interleaved manner with 256 scans for the reference and 1024 for the cross-peak experiments at 20.0, 31.1 and $40.5{ }^{\circ} \mathrm{C}$. At $50.2{ }^{\circ} \mathrm{C}, 256$ and 1258 scans were used for the reference and cross-peak experiments, respectively. In addition, at $31.1{ }^{\circ} \mathrm{C}$ the experiments were repeated with 256 and 718 scans for the reference and cross-peak experiments, respectively. The data reported for this temperature represent the average 
of the two data sets. The total acquisition time was $3.5-5$ days at each temperature. $512 \times 50$ complex points were collected with a spectral width of 14 and 25 ppm in the ${ }^{1} \mathrm{H}$ and ${ }^{15} \mathrm{~N}$ dimensions, respectively. The data were processed using the NMRpipe program [43]. The signal intensities were obtained as peak heights using the nlinLS procedure of the NMRpipe package with Gaussian line shapes and peak half-widths constrained to $2 \mathrm{~Hz}$ in each dimension. The errors were obtained from the noise in the reference and cross-peak experiments using the error propagation method. The comparison of the two data sets at $31.1{ }^{\circ} \mathrm{C}$ indicated that the experimental errors fell within the range obtained from the noise levels. The angles were taken from either NMR or X-ray structures $[37,38]$.

\subsection{Statistical Analysis}

\subsubsection{Statistical Analysis of $\tilde{r}_{u v}^{\exp }$ Values}

Because of the low sensitivity of the experiments, the data have large uncertainties. Below we present various statistical tests intended to check the significance of the data.

The values of $\tilde{r}_{u v}^{\exp }$ obtained at $50{ }^{\circ} \mathrm{C}$ were compared to $\tilde{r}_{u v}^{\exp }$ values obtained at 20,31 , and $40{ }^{\circ} \mathrm{C}$

$$
\Delta \tilde{r}_{u \nu}^{\exp }(T)=\tilde{r}_{u v}^{\exp }\left(T=50^{\circ} \mathrm{C}\right)-\tilde{r}_{u \nu}^{\exp }(T) .
$$

Data sets with angles obtained from NMR structure were analyzed separately from those that used X-ray angles. Paired statistical tests that analyze the differences in $\tilde{r}_{u \nu}^{\text {exp }}$ values (for a given residue) between each set of pairs were performed. The goal was to see whether the data at $50{ }^{\circ} \mathrm{C}$ are significantly different from the data at the other temperatures. The distribution of the differences including the means and medians are shown in Fig. 5. The $p$ values, i.e., the probabilities that the data are observed by chance, based on the null hypothesis that the mean or median differences are zero, are shown in Table 3. As all $p$ values for both parametric t-test and nonparametric Wilcoxon test are smaller than 0.05 , we conclude that the mean and median of $\Delta \tilde{r}_{u v}^{\exp }(T)$ are both significantly negative. Several statistical tests (one-way ANOVA test, Dunnett's test) showed that only $\tilde{r}_{u \nu}^{\exp }\left(T=50^{\circ} \mathrm{C}\right)$ is significantly different from the others. The statistical analysis clearly indicates that the order parameters for slow motions at $50{ }^{\circ} \mathrm{C}$ are significantly lower than one.

\subsubsection{Exclusion of Outliers}

The goal is to see whether the procedure of excluding outliers is legitimate. The values of $\tilde{r}_{u v}^{\exp }$ that were greater than 1.5 , lower than zero, or had experimental errors greater than 0.5 were excluded from the analysis. The median value for each data set (four data sets for the X-ray angles and four for NMR angles) was compared to the median value for the data set with the outliers removed 

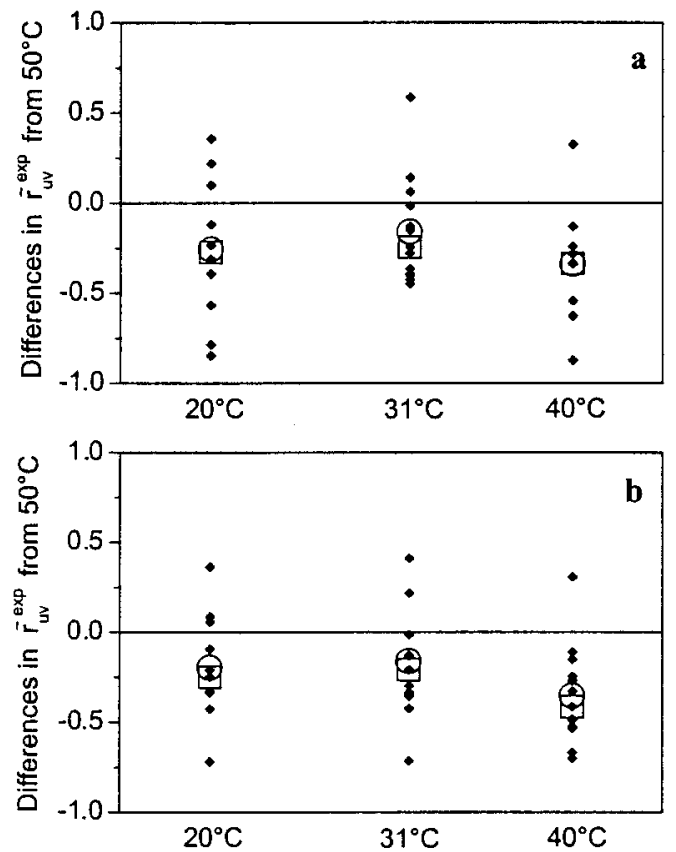

Fig. 5. Distributions of the $\Delta \vec{r}_{u v}^{\exp }(T)$ values obtained at $20,3 \mathrm{l}$, and $40{ }^{\circ} \mathrm{C}$ for a given residue obtained using either the angles from the NMR structure (a) or X-ray structure (b). Means and medians of $\Delta \tilde{r}_{u \nu}^{\exp }(T)$ are shown as $O$ and $\square$, respectively.

using a nonparametric Mann-Whitney test. The $p$ values ranged from 0.3 to 0.9 . This test allows one to reject the hypothesis that the exclusion of the outliers introduced a bias into median values for each of the eight data sets tested.

\subsubsection{Paired Tests}

Analyses were performed on the data sets with the outliers removed. The values of $\Delta \tilde{r}_{u v}^{\exp }(T)$ were compared for $T=20,31$, and $40^{\circ} \mathrm{C}$. Data sets with angles ob-

Table 3. The $p$ values from the statistical analysis of $\tilde{r}_{u v}^{\exp }$ values obtained at $50^{\circ} \mathrm{C}$ relative to $\tilde{r}_{u v}^{\exp }$ obtained at comparison temperatures and calculated either for angles obtained from NMR or X-ray structures.

\begin{tabular}{|c|c|c|c|c|c|c|}
\hline \multirow[t]{3}{*}{ Test } & \multicolumn{6}{|c|}{ Value of $p$ for difference of $\tilde{r}_{u v}^{\exp }$ at $50^{\circ} \mathrm{C}$ from that at: } \\
\hline & \multicolumn{2}{|c|}{$20^{\circ} \mathrm{C}$} & \multicolumn{2}{|c|}{$31{ }^{\circ} \mathrm{C}$} & \multicolumn{2}{|c|}{$40^{\circ} \mathrm{C}$} \\
\hline & NMR & X-ray & NMR & $\mathrm{X}$-ray & NMR & $X$-ray \\
\hline$t$-Test & 0.036 & 0.025 & 0.037 & 0.040 & 0.008 & 0.0002 \\
\hline Wilcoxon test & 0.04 & 0.03 & 0.03 & 0.04 & 0.01 & 0.0002 \\
\hline
\end{tabular}


tained from the NMR structure were analyzed separately from those that used X-ray angles. Prism4 statistical software was used (www.graphpad.com/prism/Prism.htm). Two statistical tests were performed. A one-sided paired t-test calculates the difference between each set of pairs and analyzes the list of differences based on the assumption that the differences in the entire population follow a Gaussian distribution. Note that while the assumption of the normal distribution for the $\tilde{r}_{u v}^{\exp }$ values themselves is not valid, it is reasonable to assume the normal distribution for the $\Delta \tilde{r}_{u v}^{\exp }(T)$ values. This test compares the means of two populations. The one-sided test is appropriate since we expect the mean at $50{ }^{\circ} \mathrm{C}$ to be the same or lower and the differences to be equal or less than zero. The reason for this is that we are testing the hypothesis whether or not slow motions are more pronounced at higher temperatures. The $p$ value answers the question: if the change in temperature really had no effect, what is the chance that a random sampling would result in an average effect as far from zero (or lower) as observed in this experiment? A nonparametric Wilcoxon test calculates the difference between each set of pairs and analyzes that list of differences, without invoking any assumptions on the distributional properties of these differences. The paired Wilcoxon test ranks the absolute values of the differences between the paired data in two samples and calculates statistics on the number of negative and positive differences. This test compares the medians of two populations. The $p$ value answers the question: if the median of the differences taken in the entire population is zero, what is the chance that a random sampling would result in a median as far from zero (or lower) as observed in this experiment?

\subsubsection{One-Way ANOVA and Dunnett's Test}

The distributions of the differences $\Delta \tilde{r}_{u \nu}^{\exp }(T)$ obtained with $T=20,31,40{ }^{\circ} \mathrm{C}$ were analyzed by a one-way ANOVA test using JMP software (www.jmp.com/). The one-way ANOVA test compares the means of three or more groups, assuming that data are sampled from Gaussian populations. The overall $p$ value answers the question: if the populations really have the same mean, what is the chance that a random sampling would result in means at least as far apart from one another as one observes in this experiment? The distribution of $\Delta \tilde{r}_{u v}^{\exp }(T)$ values obtained with $T=20,31,40{ }^{\circ} \mathrm{C}$ were compared.

Dunnett's test, which is a modification of the t-test, accounts for multiple comparisons, as well as for the fact that the comparisons are interrelated. Dunnett's test compares groups relative to a chosen control group. For this analysis, $\Delta \tilde{r}_{u v}^{\exp }(T)$ values for $T=40^{\circ} \mathrm{C}$ were used as control group.

\section{Conclusions}

We investigated the temperature dependence of NMR relaxation rates for backbone ${ }^{15} \mathrm{~N}-{ }^{-} \mathrm{H}$ interactions in ${ }^{15} \mathrm{~N} /{ }^{13} \mathrm{C}$ labeled human ubiquitin in the temperature range of $20-50{ }^{\circ} \mathrm{C}$. The ${ }^{15} \mathrm{~N}$ autorelaxation data give evidence that the potential 
energy functions for the $\mathrm{N}-\mathrm{H}^{\mathrm{N}}$ bonds are not quadratic, in agreement with results for other proteins. Cross-correlation rates arising from correlated fluctuations of two $\mathrm{N}-\mathrm{H}^{\mathrm{N}}$ dipole-dipole interactions involving successive residues were obtained at four temperatures. The theory of spectral densities was reviewed for independent axially symmetric fast internal motions combined with internal motions that are slower than the overall global tumbling of the molecule. The crosscorrelated rates were analyzed using the assumption of uncorrelated axially symmetric fluctuations for the slow motions. The results suggest the presence of slow internal motions at $50{ }^{\circ} \mathrm{C}$. Deviations from the assumptions of uncorrelated and axially symmetric slow internal motions are discussed, as well as the use of NMR or X-ray structures for the determination of the angles between various interactions that enter into the spectral densities. More sensitive cross-correlation experiments could provide useful constraints on the form of the potentials of correlated fluctuations.

\section{Acknowledgements}

We are grateful to Sapna Ravindranathan for help with the cross-correlation experiments and to Daniel Abergel for helpful discussions. This work has been supported by the Centre National de la Recherche Scientifique of France and the Fonds National de la Recherche Scientifique of Switzerland.

\section{References}

1. Palmer A.G.: Annu. Rev. Biophys. Biomol. Struct. 30, 129-155 (2001)

2. Ishima R., Torchia D.A.: Nat. Struct. Biol. 7, 740-743 (2000)

3. Palmer A.G., McDermott A.J.: Phys. Chem. 199, 13293-13310 (1996)

4. Daragan V.A., Mayo K.H.: Prog. Nucl. Magn. Reson. Spectrosc. 32, 63105 (1997)

5. Frueh D.: Prog. Nucl. Magn. Reson. Spectrosc. 41, 305-324 (2003)

6. Brutscher B.: Concepts Magn. Reson. 12, 207-234 (2000)

7. Daragan V.A., Mayo K.H.: J. Magn. Reson. 100, 8378-8388 (1996)

8. Daragan V.A., Mayo K.H.: J. Phys. Chem. B 103, 6829-6834 (1999)

9. Felli I.C., Desvaux H., Bodenhausen G.: J. Biomol. NMR 12, 509-521 (1998)

10. Pelupessy P., Ravindranathan S., Bodenhausen G.: J. Biomol. NMR 25, 265-280 (2003)

11. Jaenicke R.: Proc. Natl. Acad. Sci. USA 96, 13674-13678 (1999)

12. Detschman W.A., Dahlquist F.W.: Biochemistry 40, 13107-13113 (2001)

13. Bracken C., Carr P.A., Cavanagh J., Palmer A.G.: J. Mol. Biol. 285, 2133-2146 (1999)

14. Seewald M.J., Pichumani K., Stowell C., Tibbals B.V., Regan L., Stone M.J.: Protein Sci. 9, $1177-$ $1193(2000)$

15. Ornuchin J.N., Luthey-Schulten Z., Wolyness P.G.: Annu. Rev. Phys. Chem. 48, 545-600 (1997)

16. Vugmeyster L., Trott O., McKnight C.J., Raleigh D.P., Palmer A.G.: J. Mol. Biol. 320, 841-854 (2002)

17. Mandel A.M., Akke M., Palmer A.G.: Biochemistry 35, 16009-16023 (1996)

18. Cordier F., Grzesiek S.: J. Mol. Biol. 715, 739-752 (2002)

19. Yang D., Kay L.E.: J. Mol. Biol. 263, 369-382 (1996)

20. Yang D., Mok Y.K., Forman-Kay J.D., Farrow N.A., Kay L.E.: J. Mol. Biol. 272, 790-804 (1996)

21. Idiyatulin D., Nesmelova I., Daragan V.A., Mayo K.H.: J. Mol. Biol. 325, 149-162 (2003)

22. Evenas J., Forseen S., Malmendal A., Akke M.: J. Mol. Biol. 289, 603-617 (1999)

23. Akke M., Bruschweiler R., Palmer A.G.: J. Am. Chem. Soc. 115, 9832-9833 (1993) 
24. Wang T., Cai S., Zuiderweg E.R.P.: J. Am. Chem. Soc. 125, 8639-8643 (2003)

25. Krizova H., Zidek L., Stone M.J., Novotny M.V., Sklenar V.: J. Biomol. NMR 28, 369-384 (2004)

26. Farrow N.A., Muhandiram R., Singer A.U., Kay C.M., Gish G., Shoelson S.E., Pawson T., FormanKay J.D., Kay L.E.: Biochemistry 33, 5984-6003 (1994)

27. Lipari G., Szabo A.J.: J. Am. Chem. Soc. 104, 4546-4559 (1982)

28. Clore M.G., Szabo A., Bax A., Kay L.E., Driscoll P.C., Gronenborn A.M.: J. Am. Chem. Soc. 112, 4989-4991 (1990)

29. Wist J., Frueh D., Tolman J.R., Bodenhausen G.: J. Biomol. NMR 28, 263-272 (2004)

30. Wintrode P.L., Makhatadze G.I., Privalov P.L.: Proteins 18, 246-253 (1994)

31. Vugmeyster L., Pelupessy P., Vugmeister B.E., Abergel D., Bodenhausen G.: C. R. Phys. 5, 377$386(2004)$

32. Tjandra N., Feller S.E., Pastor R.W., Bax A.: J. Am. Chem. Soc. 117, 12562-12566 (1995)

33. Fushman D., Tjandra N., Cowburn D.: J. Am. Chem. Soc. 121, 8577-8582 (1999)

34. Lee A.L., Wand A.J.: J. Biomol. NMR 13, 101-112 (1999)

35. Lee L.K., Rance M., Chazin W.J., Paimer A.G.: J. Biomol. NMR 9, 287-298 (1997)

36. Peti W., Meiler J., Bruschweiler R., Griesinger C.: J. Am. Chem. Soc. 124, 5822-5833 (2002)

37. Vijay-Kumar S., Bugg C.E., Cook C.J.: J. Mol. Biol. 194, 531-534 (1987)

38. Cornilescu G., Marquardt J.L., Ottiger M., Bax A.: J. Am. Chem. Soc. 120, 6836-6837 (1998)

39. Kroenke C.D., Rance M., Palmer A.G.: J. Am. Chem. Soc. 121, 10119-10125 (1999)

40. Marion D., Ikura M., Tschudin T., Bax A.: J. Magn. Reson. 48, 286-292 (1989)

41. Cavanagh J., Palmer A.G., Wright P.E., Rance M.: J. Magn. Reson. 91, 429-436 (1991)

42. Kay L.E., Keifer P., Saarinen T.: J. Am. Chem. Soc. 114, 10663-10665 (1992)

43. Delaglio F., Grzesiek S., Vuister G.W., Zhu G., Pfeifer J., Bax A.: J. Biomol. NMR 6, 277-293 (1995)

44. Mosteller F., Tukey J.W.: Data Analysis and Regression: A Second Course in Statistics. Reading: Addison-Wesley 1977.

45. Mandel A.M., Akke M., Palmer A.G.: J. Mol. Biol. 246, 144-163 (1995)

Authors' address: Geoffrey Bodenhausen, Département de Chimie, Associé au CNRS, Ecole Normale Supérieure, 24 rue Lhomond, 75231 Paris cedex 05, France 